\title{
Simultaneous Production and Sustainable Eutectic Mixture Based Purification of Narringinase With Bacillus Amyloliquefaciens by Valorization of Tofu Wastewater
}

Harishbabu Balaraman

SASTRA University

Purushotaman C

SASTRA University

Chandramouliswaran $\mathrm{K}$

SASTRA University

Senthil Kumar Rathnasamy ( $\nabla$ senthilrathna@sastra.ac.in )

SASTRA University

Research Article

Keywords:

Posted Date: January 31st, 2022

DOI: https://doi.org/10.21203/rs.3.rs-1271183/v1

License: (c) (i) This work is licensed under a Creative Commons Attribution 4.0 International License.

Read Full License 


\section{Abstract}

Sustainable one-pot production and purification of naringinase using natural deep eutectic solvent based extractive fermentation is being executed in the current investigation. Tofu wastewater was used as alternative low-cost media for sustainable production of naringinase. Five natural deep eutectic solvents were prepared and their physicochemical properties were determined as a function of temperature. Optimization of essential variables in extractive fermentation for determining effective concentration of NADES at $(74.5 \% \mathrm{w} / \mathrm{w}), \mathrm{Na}_{2} \mathrm{SO}_{4}(15 \% \mathrm{w} / \mathrm{v})$ and tofu wastewater $(1.5 \% \mathrm{w} / \mathrm{w})$ to achieve optimal naringinase yield of $(249.6 \mathrm{U} / \mathrm{ml})$. Scale up of naringinase production with a $3 \mathrm{~L}$ custom made desktop bioreactor was accomplished and effective regeneration of NADES was established. Quantification of the resulting active enzyme was done by size exclusion chromatography $(736.85 \mathrm{U} / \mathrm{mg})$. Ultrapure enzyme fraction was obtained with anion exchange chromatography yielding maximum purity of (63.2) and specific enzyme activity of $(3516 \mathrm{U} / \mathrm{mg})$. The in-vitro debittering activity of resulting ultrapure naringinase was determined with grape juice.

\section{Introduction}

Naringin (4,5,7-Trihydroxyflavanone 7-rhamnoglucoside) is an commercially important flavonoid responsible for causing acerbity in citrus fruits ${ }^{1}$. It is rich in antibiotics and is widely used for the biotransformation of steroids ${ }^{2}$. Naringinase is a microbial enzyme that acts on naringin converting it into rhamnose and prunin, which is further hydrolysed to yield naringenin and glucose ${ }^{3}$. Various food and beverage manufacturing industries require large quantities of naringinase for diverse applications including debittering of fruit juices ${ }^{4}$. Further, the intermediate product prunin produced during this process can inhibit acyl-CoA-cholesterol-O-acyl transferase reducing the incidence of hepatic disease ${ }^{5}$. Hydrolysis products like naringenin and rhamnose are used as starting materials in various pharmaceutical and cosmetic industries thus inherit commercial importance ${ }^{6}$. This significance of the enzyme insists on delving a sustainable approach in the production and purification of naringinase ${ }^{7}$.

Naringinase is produced in large quantities by all types of microbial species like fungi (Aspergillus spp.), bacteria (Bacillus amyloliquefaciens) and yeast ${ }^{8}$. Recent studies have been focusing on enhancing the extracellular production of this enzyme by submerged fermentation with various bacterial species ${ }^{9}$. However, the conventional method of enzyme production employing synthetic media have been observed to contribute a significant fraction of production cost making it unsuitable for industrial scale ${ }^{10}$. Substitution of these commercial media with low-cost complex agricultural residues provides a viable alternative for the sustainable production of naringinase ${ }^{11}$. Earlier investigation on the sustainable production of naringinase by employing molasses and peptone as carbon and nitrogen sources respectively has been accomplished ${ }^{12}$. In a similar investigation of naringinase production, Borkar et al have identified that introduction of orange peel waste as an inducer has substantially promoted enzyme production ${ }^{13}$. In yet another identical investigation carried out by Puri et al, optimal production of naringinase was accomplished with soybean meal as an alternative low-cost substrate ${ }^{14}$. These 
investigations have proven that exploiting agricultural and industrial by-products have a substantial influence on enhancing the sustainability of naringinase production.

The conventional method of naringinase purification involves salt induced precipitation followed by sequential chromatography. Even though this approach provides enhanced purity, the native state of the product gets affected due to the prevailing harsh environmental conditions resulting in a lower yield of active enzyme ${ }^{15}$. In an investigation accomplished by Zhu et al, ammonium sulfate precipitation followed by gel filtration and anion exchange chromatography has resulted in an overall enzyme recovery of $32 \%$ with the specific activity of 2194.2 units ${ }^{11}$. A similar investigation carried out by Chen et al have resulted in an overall naringinase yield of $7.1 \%$ with the corresponding purity fold of $17.2^{16}$. In contradiction, employing three phase partitioning for purification of naringinase have resulted in 4.4 fold enzyme purity with a maximum yield of $96 \%{ }^{17}$. From these investigations, it is conclusive that employing two (or) three phase partitioning has a positive influence on both the yield and purity of enzyme ${ }^{18}$. Extractive fermentation is one of the most advanced processes integration mechanism providing a feasible solution for the existing complications in the purification of naringinase ${ }^{19}$. In extractive fermentation, the product is recovered directly from the culture by the formation of two phase which readily infiltrates both enzyme and whole cell into the two immiscible layers respectively ${ }^{20}$. Earlier investigations on extractive fermentation have employed PEG as the major extracting agent posing several disadvantages including low product specificity and regeneration capacity attributed to their hydrophobic nature ${ }^{21}$. In modern days, sustainable alternatives like deep eutectic solvents (DES) have been employed to address the key issues of extractive fermentation ${ }^{22} 23$. Beyond encasing several advantages of being superficial and low cost in synthesis, they are task-specific and are facile for regeneration ${ }^{24}$. Earlier investigations have employed DES as a task-specific medium to purify therapeutically important enzymes like protease ${ }^{25}$ and immunoglobulins ${ }^{26}$. Therefore, these investigations have concluded the selective and task specific nature of NADES making it a suitable medium for extractive fermentation of naringinase 27,28 .

The present investigation aims at simultaneous production and purification of naringinase by extractive fermentation by administering NADES as an extraction medium. Four low-cost complex media were evaluated for the maximum yield of naringinase. Synthesis of five NADES was accomplished and their thermophysical behaviour was recorded. Screening of suitable NADES for extractive fermentation of naringinase was performed by evaluating the phase ratio and partition coefficient of individual NADES with various salts. Response surface methodology based optimisation of the concentration of NADES, $\mathrm{Na}_{2} \mathrm{SO}_{4}$ and tofu wastewater was done and the resulting naringinase yield was determined.

Quantification of the enzyme was accomplished with gel filtration chromatography and purity was enhanced with anion exchange chromatography. Kinetics of the purified enzyme was evaluated with naringin as substrate and debittering activity of ultrapure naringinase was determined in grape juice.

\section{Materials And Methods}




\subsection{Chemicals and reagents}

Benzyl trimethyl ammonium chloride, Bovine Serum Albumin and Naringin were procured from Sigmaaldrich, USA. Lactose, Maltose, Fructose, Sucrose, Xylose, Sodium Sulphate, Di-Potassium Hydrogen Phosphate and Sodium Carbonate were procured from Himedia, India. The purity of each chemical used in the current investigation is provided in Table 1.

Table 1

Source and purity of chemicals used in preparation of NADES and extractive fermentation

\begin{tabular}{|llll|}
\hline Chemical Name & $\begin{array}{l}\text { CAS Reg. } \\
\text { No. }\end{array}$ & Supplier & $\begin{array}{l}\text { Mass \% purities stated by } \\
\text { suppliers }\end{array}$ \\
\hline $\begin{array}{l}\text { Benzyl trimethyl ammonium } \\
\text { chloride (BMC) }\end{array}$ & $56-93-9$ & $\begin{array}{l}\text { Sigma- } \\
\text { aldrich }\end{array}$ & $0.99 \mathrm{w} / \mathrm{w}$ \\
\hline Bovine serum albumin & $9048-46-8$ & $\begin{array}{l}\text { Sigma- } \\
\text { aldrich }\end{array}$ & $0.99 \mathrm{w} / \mathrm{w}$ \\
\hline Naringin & $10236-47-$ & $\begin{array}{l}\text { Sigma- } \\
\text { aldrich }\end{array}$ & $0.99 \mathrm{w} / \mathrm{w}$ \\
\hline Lactose (L) & 2 & Himedia & $0.97 \mathrm{w} / \mathrm{w}$ \\
\hline Fructose (F) & $63-42-3$ & Himedia & $0.97 \mathrm{w} / \mathrm{w}$ \\
\hline Sucrose (S) & $58-49-7$ & Himedia & $0.97 \mathrm{w} / \mathrm{w}$ \\
\hline Xylose (X) & $57-50-1$ & Himedia & $0.97 \mathrm{w} / \mathrm{w}$ \\
\hline Maltose (M) & $609-06-3$ & Himedia & $0.97 \mathrm{w} / \mathrm{w}$ \\
\hline Sodium sulphate & $69-79-4$ & Himedia & $0.97 \mathrm{w} / \mathrm{w}$ \\
\hline Di-potassium Hydrogen Phospate & $7758-11-4$ & Himedia & $0.97 \mathrm{w} / \mathrm{w}$ \\
\hline Sodium carbonate & $207-838-8$ & Himedia & $0.97 \mathrm{w} / \mathrm{w}$ \\
\hline
\end{tabular}

\subsection{Sample collection and inoculum preparation}

A sterile lyophilized culture of Bacillus amyloliquifaciens was procured from Microbial Type Culture Amassment (MTCC), Chandigarh. The culture was transferred to a liquid nutrient medium supplemented with naringin $(1 \% \mathrm{w} / \mathrm{v})$ to induce naringinase production and incubated overnight at $37^{\circ} \mathrm{C}, 150 \mathrm{rpm}$. A loop full of the overnight incubated culture was then transferred to the naringin plate and a single pure colony with enhanced naringinase activity was obtained. This pure colony of $B$. amyloliquifaciens was sub-cultured and maintained on nutrient agar plates and the seed culture was sub-cultured every 30 days. 
Vegetative inoculum was prepared with the LB media ( $1 \mathrm{~g} / \mathrm{L}$ tryptone, $0.5 \mathrm{~g} / \mathrm{L}$ yeast extract, $1 \mathrm{~g} / \mathrm{L} \mathrm{NaCl})$ ( $\mathrm{pH}$ 7.0). $100 \mathrm{ml}$ of media was prepared and sterilized at $15 \mathrm{lbs} / \mathrm{in}^{2}\left(121^{\circ} \mathrm{C}\right.$ for 15 minutes). The flask containing sterile media was cooled to room temperature and a loop full of culture from the seed culture plate was inoculated and maintained at 200 rpm, 37 C for 24 hours.

\subsection{Screening of complex media for naringinase production}

A variety of low-cost industrial and agricultural by-products such as Cane molasses, tofu wastewater, Corn steep liquor were chosen as candidates for naringinase production. All these complex media sources were collected from different localities in and around Salem. Cane molasses was collected from a sugarcane industry at Mettur, tofu wastewater was collected from soy meal processing unit at Attur, Corn steep liquor was collected from a cottage industry located in Thiruchengode. The collected sources were pre-filtered with $0.2 \mu \mathrm{M}$ cheesecloth, sterilized and preserved at $-4^{\circ} \mathrm{C}$ for further use.

$100 \mathrm{ml}$ of the fermentation medium was prepared by mixing $(15 \% \mathrm{w} / \mathrm{v})$ of maltose, $(10 \% \mathrm{w} / \mathrm{v})$ of $\mathrm{NaCl}$, $(20 \% \mathrm{w} / \mathrm{v})$ of $\mathrm{NH}_{4} \mathrm{HPO}_{4}$ and $(7 \% \mathrm{w} / \mathrm{v})$ of naringin to the diluted complex media $(0.5 \% \mathrm{v} / \mathrm{v})$ along with trace amounts of other micronutrients. The prepared media was sterilized at $15 \mathrm{psi}$ and $121^{\circ} \mathrm{C}$ and inoculated with $1 \mathrm{ml}$ of vegetative seed culture. The inoculated medium was incubated at $150 \mathrm{rpm}$ in a refrigerated shaker incubator (REMI, India) until it reaches the late log phase.

\subsection{Synthesis and thermophysical characterization of NADES}

Five natural deep eutectic solvents (NADES) were synthesized by mixing Benzyl trimethyl ammonium chloride with various carbohydrate molecules (Lactose, Fructose, Xylose, Maltose, Sucrose) in molar ratio of $1: 1$. The mixture was heated in an oil bath at $80^{\circ} \mathrm{C}$ for 2 hours until it attained homogeneity. The NADES thus formed was cooled to room temperature and its stability was monitored for 48 hours at room temperature. $\mathrm{H}^{1} \mathrm{NMR}$ of the synthesized NADES was determined with $300 \mathrm{MHz}$ BRUKER AVANCE II spectrometer (Bruker Biospin, Switzerland) equipped with $5 \mathrm{~mm}$ BBO probe. The sample (8 mg approx.) was dissolved in $500 \mu \mathrm{l}$ of $\mathrm{D}_{2} \mathrm{O}$ in the quartz tube provided and ultra-sonicated. The resonance of sample was recorded at $298.15 \mathrm{~K}$ and processed using pulse sequence library Topspin 3.2. (Bruker Biospin, Switzerland)

Thermophysical behavior of all NADES was recorded as a function of temperature in the temperature interval of $(273-323 \mathrm{~K})$. Density of all NADES was measured with Rudolf digital density meter DDM 2910 (Rudolf, USA). Viscosity of the eutectic mixture was determined with Brookefield LV II+ digital viscometer (Brookefield, USA). Refractive index of the samples was determined with ATAGO MASTER-PM portable refractometer with sodium D1 line (Atago, Japan). All the instruments were pre-calibrated with deionized water and respective standards before measurement.

\subsection{Extractive bioconversion for naringinase production}


Extractive fermentation was carried out in a $250 \mathrm{ml}$ Erlenmeyer flask using the five different sterile NADES $(80 \% \mathrm{v} / \mathrm{v})$ under aseptic conditions. For efficient product recovery, the solvent was added in the time interval of 12-14 hours due to the optimal production of naringinase. Post addition of NADES, the mixture was titrated with the corresponding salt solution $(20 \% \mathrm{w} / \mathrm{v})$ under continuous agitation until the solution turns milky. This heterogeneous mixture was incubated for $2 \mathrm{~h}$ at room temperature for the formation of two distinct phases. After a clear boundary was visible between the two phases, the volume of the individual phase and its corresponding naringinase activity was measured by the method mentioned by Chen et $\mathrm{al}^{16}$.

Selection of NADES and salt combination that has optimal phase formation capacity is a mandatory step towards enabling efficient extractive fermentation. Various salts such as $\left(\mathrm{Na}_{2} \mathrm{SO}_{4}, \mathrm{Na}_{2} \mathrm{CO}_{3}\right.$ and $\left.\mathrm{K}_{2} \mathrm{HPO}_{4}\right)$ were screened for their respective phase formation capacity in combination with all five NADES. The effect of salt choice on phase formation was evaluated by calculating the phase ratio for all combinations with the following equation.

$$
P=\frac{V_{e}}{V_{r}}(1)
$$

Where $V_{e}$ is the volume of the top phase and $V_{r}$ is the volume of the bottom phase in the ext.

The partition coefficient of the extractive fermentation system needs to be significantly high enough to enable the selective concentration of the product in the NADES phase. Evaluation of partition coefficient of all NADES results in the identification of the suitable solvent acting most selective towards naringinase extraction. Each NADES mixture was titrated against $\mathrm{Na}_{2} \mathrm{SO}_{4}$ and the amount of enzyme recovered in the top phase was determined. The partition coefficient of the system was determined with the following equation.

$$
K=\frac{C_{e}}{C_{r}}(2)
$$

Where $C_{e}$ indicates the extractant phase naringinase concentration and $C_{r}$ indicates the concentration of naringinase in the raffinate phase. The mean values of standard analyte were estimated in triplicates and represented along with standard deviation.

\subsection{Determination of specific enzyme activity using Narringin}

Naringinase activity was determined by the modified Davis method ${ }^{16}$. To the $1 \mathrm{ml}$ of $0.1 \%$ naringin solution prepared with acetate buffer $(\mathrm{pH} 4.2), 200 \mu$ l of culture filtrate was added and incubated at $60^{\circ} \mathrm{C}$ for 60 minutes. $0.1 \mathrm{ml}$ of aliquot was withdrawn from the mixture and titrated with $5 \mathrm{ml}$ of $90 \%$ diethylene glycol and $10 \mu \mathrm{l}$ of $4 \mathrm{~N} \mathrm{NaOH}$. The residual naringin present in the sample yields yellow and the intensity was quantified at $420 \mathrm{~nm}^{29}$. Enzyme activity was calculated utilizing pristine naringin as the standard. 
Enzyme activity $=\frac{\text { Absorbance at } 420 \mathrm{~nm}\left(\frac{\mathrm{mg}}{\mathrm{ml}}\right) \times \text { Reaction volume }(\mathrm{ml}) \times 10^{3}\left(\frac{\mu \mathrm{gmol}}{\mathrm{mgmol}}\right)}{}$

Equivalent weight $\left(\frac{\mathrm{mg}}{\mathrm{mgmol}}\right) \times$ reaction time $(\mathrm{min})$

Specific Enzyme activity $=\frac{\text { Enzyme activity }(\mathrm{U})}{\text { Total protein in culture medium }(\mathrm{g})}$

\subsection{Optimization of complex medium for Naringinase production}

The influence of each essential variable over the production of naringinase was optimized using response surface methodology. The statistical evaluation was done with a three level central composite design (CCD) (Design Expert, v10.1 Stat-ease, Minneapolis, USA) for three variables. The concentration of NADES $(65-80 \% \mathrm{w} / \mathrm{w}), \mathrm{Na}_{2} \mathrm{SO}_{4}(10-20 \% \mathrm{w} / \mathrm{v})$ and tofu wastewater $(0.5-2.5 \% \mathrm{v} / \mathrm{v})$ were chosen as variable and the corresponding yield of naringinase was considered as the response variable. A cluster of 32 runs including 6 centre points, axial points and factorial points was carried out. In a batch experiment, $100 \mathrm{ml}$ of the grown culture was titrated with the corresponding amount of NADES and $\mathrm{Na}_{2} \mathrm{SO}_{4}$ until cloud point formation. The extract phase of the system post equilibrium was recovered and the corresponding amount of naringinase was determined by the specific enzyme assay as previously described.

\subsection{Scale-up of extractive fermentation in desktop custom bioreactor}

Scaling up of extractive bioconversion was performed with a $3 \mathrm{~L}$ custom made extractive fermentation vessel with $1.8 \mathrm{~L}$ working volume. Initially, the reactor was sterilized by autoclaving at $121^{\circ} \mathrm{C}$ for $2 \mathrm{~h}$. The addition of sterile media and phase forming components (BMC:X and $\mathrm{Na}_{2} \mathrm{SO}_{4}$ ) was done inside a laminar airflow chamber to avoid external contamination. For a batch process, $1.5 \mathrm{~L}$ of fresh sterile soy milk media was prepared in the previously determined optimal concentration of $1.5 \% \mathrm{v} / \mathrm{v}$ and added to the bioreactor. Fermentation was initiated with $5 \% \mathrm{w} / \mathrm{v}$ inoculum of $B$. amyloliquefaciens at $30^{\circ} \mathrm{C}$ and $\mathrm{pH}$ 6.5 with the corresponding dissolved oxygen concentration of $50 \% \mathrm{v} / \mathrm{v}$. Post the batch hour $(16 \mathrm{~h})$, the phase forming agents (NADES and salt) were added in predetermined optimized concentration along with agitation until cloud point and the media was left undisturbed for 30 minutes for distinct phase formation. The top phase was recovered in sterile condition and evaluation of its corresponding naringinase activity was accomplished.

\subsection{Sequential batch recovery of naringinase from fermentation media}


Sequential batch recovery of naringinase was accomplished by removing the top phase containing naringinase and supplementing the raffinate phase rich in whole cell with the fresh media $(5 \% \mathrm{v} / \mathrm{v})$ in a fed batch mode operation. As observed in batch mode, phase forming components were supplemented during the harvesting phase and the top phase rich in naringinase was obtained.

\subsection{Regeneration and reuse of NADES for sequential extractive fermentation}

The NADES rich top phase along with the naringinase enzyme obtained from extractive fermentation was removed and stored in a sterile container. The NADES used in the concentration of naringinase needs to be recovered and reused for sequential batch extractive fermentation. This was accomplished by treating the concentrated top phase with kosmotropic salts like $\mathrm{NaCl}(15 \% \mathrm{w} / \mathrm{v})$. The agitation was continued until the solution becomes homogenous. The mixture was then left undisturbed for $2 \mathrm{~h}$ until two distinct phases were formed. The NADES rich top phase was collected in a sterile container and recycled along with the fresh NADES supplied from the reservoir. The amount of NADES recovered was calculated by the following equation:

$$
Y_{D E S}(\%)=\frac{V_{R}(m l)}{V_{0}(m l)} * 100(5)
$$

Where, $Y_{D E S}$ signifies the yield of DES recovery, $V_{R}$ is the volume of DES recovered from top phase, $V_{0}$ is the volume of DES added for extractive fermentation

\subsection{Enzyme kinetics}

The maximum rate of enzyme activity $\left(\mathrm{V}_{\text {max }}\right)$ and the minimum concentration of substrate $\left(\mathrm{K}_{\mathrm{m}}\right)$ required to saturate the enzyme was determined by calculating the rate of reaction at increasing substrate concentration ${ }^{30}$. Naringin was used as substrate and was taken at different concentration for determining the optimal conditions of enzyme activity ${ }^{31}$. The following Line-weaver Burk equation was used to calculate the apparent $\mathrm{K}_{\mathrm{m}}$ and $\mathrm{V}_{\max }$ values.

$$
[S] / V_{o}=1 / V_{\max }[S]+K_{m} / V_{\max }
$$

Where [S] denotes Naringin concentration, $\mathrm{V}_{0}$ is the initial rate of reaction, $\mathrm{V}_{\mathrm{max}}$ is the maximum rate of reaction, $K_{m}$ is the minimum concentration of substrate required to achieve $1 / 2\left[V_{\text {max }}\right]$.

Using the $\mathrm{K}_{\mathrm{m}}$ and $\mathrm{V}_{\mathrm{max}}$, the corresponding turn over constant $\left(\mathrm{K}_{\mathrm{cat}}\right)$ and the catalytic efficiency of the enzyme $\left(\mathrm{K}_{\mathrm{cat}} / \mathrm{K}_{\mathrm{m}}\right)$ can be determined. The turn over constant $\left(\mathrm{K}_{\mathrm{cat}}\right)$ is denoted by $\left[\mathrm{V}_{\mathrm{max}} /\left[\mathrm{E}_{0}\right]\right]$, where $\left[\mathrm{E}_{0}\right]$ is concentration of active enzyme. 


\subsection{Quantification of naringinase by gel filtration chromatography}

Gel filtration chromatography based quantification of naringinase recovered from the extractive fermentation was accomplished. A size exclusion column (Sephadex G-15, AKTA prime plus, GE, USA) of $5 \mathrm{ml}$ volume was used for the fractionation of the enzyme. The column was equilibrated with $(20 \mathrm{mM}$ phosphate buffer, $\mathrm{pH} 7$ ) before the sample injection to set the baseline at zero ${ }^{32}$. The flow rate throughout the process is maintained constant at $1 \mathrm{ml} / \mathrm{min}$. The enzyme fraction concentrated from back extraction was pre-filtered with a syringe filter of $0.22 \mu \mathrm{m}$ pore size (Sigma-Aldrich, USA). The sample was then eluted with the elution buffer ( $20 \mathrm{mM}$ phosphate buffer, $0.1 \mathrm{M} \mathrm{NaCl}, \mathrm{pH}$ 7) and the eluents corresponding to the peak observed with the UV detector were collected. The retention volume is calculated as the volume of the mobile phase passed through the column to mobilize naringinase from its entry into the column until the detector detects the compound. The retention volume is calculated by the following equation.

$$
V_{R}=t_{R} \times F(7)
$$

The number of theoretical plates were evaluated to determine the quantitative efficiency of the separation. The number of theoretical plates for the present system of sephadex and buffer is given by the following equation

$$
n=16\left(\frac{t_{r}}{w_{b}}\right)^{2}
$$

The resolution $\left(R_{s}\right)$ between two successive peaks is observed to be a measure of separation. Resolution factor can be calculated by

$$
R_{s}=2\left(\frac{t_{R 2}-t_{R 1}}{w_{b 1}+w_{b 2}}\right)(9)
$$

\subsection{Purification of naringinase by anion exchange chromatography}

The purity fold of the fractions purified by gel filtration chromatography was exponentially increased by performing anion exchange chromatography. The DEAE-sepharose anion exchange column with $5 \mathrm{ml}$ bed volume was chosen to enrich the purity of naringinase. The column was set to zero baseline with phosphate buffer $(20 \mathrm{mM}, \mathrm{pH} 5.5)$ at a flow rate of $1 \mathrm{ml} / \mathrm{min}^{33}$. Further, the sample was injected into the sample injector which carries the sample along with binding buffer into the column. Molecules that do 
not have an affinity with the matrix were eluted along with the binding buffer. The protein fractions bound to the matrix was eluted with elution buffer $(20 \mathrm{mM}$ citrate buffer, $\mathrm{pH} 5.5,1 \mathrm{M} \mathrm{NaCl})$ in the increasing gradient of affinity. The absorbance of the ultrapure naringinase enzyme was determined with the UV detector and the corresponding fraction was collected with the fraction collector.

\subsection{Debittering activity of naringinase in grape fruit juice}

Debittering activity of ultrapure naringinase was determined using fresh grape pulp. Initially, fresh grapefruit purchased from the local market was peeled and seeds were removed. The pulp was squeezed with mortar and pestle and the crude juice obtained was centrifuged ( $8000 \mathrm{rpm}, 30 \mathrm{~min}$ ) to obtain clarified juice. $5 \mathrm{ml}$ of grape juice was incubated with $50 \mu \mathrm{l}$ of ultrapure enzyme for 20 minutes at $37^{\circ} \mathrm{C}$. Post incubation, the enzyme was inactivated by heating the mixture at $100^{\circ} \mathrm{C}$ for 5 minutes and cooled to room temperature. The obtained product is centrifuged (8000 rpm, $20 \mathrm{~min}$ ) and the clear supernatant was filtered through a syringe filter. The enzyme-treated fraction was injected into the $\mathrm{C} 18$ column for evaluation of naringin and limonin remaining in the juice. The initial amount of naringin present in the juice was quantified with the clarified juice before enzyme addition. Naringin and limonin present in the grapefruit juice before and after naringinase addition was quantified by high pressure liquid chromatography LC20AD (Shimadzu, Japan) operated at 207 and $283 \mathrm{~nm}$ respectively as described by Ribero et al $^{34}$.

\section{Result And Discussion}

\subsection{Thermophysical and spectral characterization of NADES}

The $\mathrm{H}^{1}$ NMR spectroscopy of all NADES is represented in Fig S1-S5. The results reveal a close interaction between HBA and HBD molecule denoted by various new peaks occurring in the spectrum. Additionally, displacement of peaks denoting Benzyl trimethyl ammonium chloride and carbohydrates occurs which signifies a stable positive non-covalent interaction between the participating molecules ${ }^{35}$. Therefore, synthesis of all NADES was accomplished and confirmed with $\mathrm{H}^{1} \mathrm{NMR}$ analysis.

\subsubsection{Density of NADES as a function of temperature}

The density of all NADES synthesized by mixing benzyl trimethyl ammonium chloride in combination with various carbohydrates was measured in the temperature interval of $(293-323 \mathrm{~K})$. The observation indicates that the density of all NADES decrease identically and form a linear relationship with temperature (Fig. 1(A)). This is due to the destabilization of non-covalent interactions between the hydrogen bond donor and the acceptor in the eutectic mixture ${ }^{36}$. NADES formed with the lower molecular weight carbohydrates (lactose) is observed to have lower density while the higher molecular weight counterparts form a denser mixture. The order of density for all the NADES studies is observed as (BMC:L 
$<\mathrm{BMC}: \mathrm{F}<\mathrm{BMC}: \mathrm{X}<\mathrm{BMC}: \mathrm{M}<\mathrm{BMC}: \mathrm{S}$ ) which corresponds to the molecular weight of hydrogen bond donor in ascending order. Elevated density signifies the close interaction of participating molecules hence less free volume for accommodating the product. Therefore, NADES with higher density has a moderate partition coefficient for naringinase in extractive fermentation. On contradictory, NADES with low density remains unsuitable for extractive fermentation due to its low salting out capability thus inefficient in extracting protein molecule to top phase ${ }^{37}$. Hence, NADES with moderate density show more affinity towards phase formation and acts selectively in the extraction of naringinase to the NADES rich top phase. The corresponding values of density of all NADES are related to the temperature with the following equation

$$
\rho=X_{1} T+X_{2}(10)
$$

Where $\rho$ is density denoted by $\left(\mathrm{kg} \mathrm{m}^{-3}\right), \mathrm{T}$ is the temperature in Kelvin and $\mathrm{X}_{1}, \mathrm{X}_{2}$ denote the linearity coefficients whose values are provided in Table 2.

Table 2

Temperature relative parameters evaluated for Density $\left(X_{1}\right.$ and $\left.X_{2}\right)$, Viscosity $\left(\mu_{0}\right.$ and $\mathrm{K}^{0}$ ) and Refractive Index ( $\mathrm{s}$ and $\mathrm{u}$ ) respectively.

\begin{tabular}{|c|c|c|c|c|c|c|}
\hline \multirow[t]{2}{*}{ NADES } & \multicolumn{2}{|c|}{ Density $\left(\mathrm{g} \mathrm{cm}^{-3}\right)$} & \multicolumn{2}{|c|}{ Viscosity (mPa.s) } & \multicolumn{2}{|c|}{ Refractive Index $\left(n_{D}\right)$} \\
\hline & $x_{1}$ & $X_{2} \cdot 10^{-4}$ & $\mu_{0}$ & $E \mu / R$ & s & u \\
\hline BMC:L & 1.085 & -4.24 & $7.92 \times 10^{-7}$ & -2423.28 & 123.71 & -0.287 \\
\hline BMC:F & 1.076 & -3.41 & $8.2 \times 10^{-13}$ & -1876.52 & 138.24 & -0.573 \\
\hline BMC:X & 1.107 & -3.02 & $6.42 \times 10^{-20}$ & -1432.86 & 151.37 & -0.684 \\
\hline BMC:M & 1.112 & -5.12 & $8.72 \times 10^{-3}$ & -2127.6 & 173.87 & -0.769 \\
\hline BMC:S & 1.124 & -6.23 & $9.3 \times 10^{-9}$ & -3932.72 & 186.21 & -0.912 \\
\hline
\end{tabular}

\subsubsection{Thermophysical delineation of viscosity for NADES}

All synthesized NADES were exposed to viscosity measurement in temperature interval of (298 - $323 \mathrm{~K})$. Notable is the identical non-linear reduction of viscosity for all NADES as a function of temperature (Fig. 1(B)). This could be due to the increased entropy of participating molecules at higher temperature elevating its fluidity ${ }^{38}$. The order of viscosity is similar to the ascending order of density and is dependent on the intensity of side chain interactions of both HBA and HBD. Higher viscosity of NADES corresponds to lower degree of ionization hence lower free energy for transport of target molecules to extraction phase. NADES with low viscosity have increased fluidity and low cohesive force and are incompetent to form two phase in extractive fermentation ${ }^{39}$. Therefore, NADES with moderate viscosity acts as better 
choice for formation of two phase in extractive fermentation of naringinase. The relativity of temperature with viscosity of NADES is represented by following equation

$$
\eta=\eta_{0} e^{-\frac{E_{\eta}}{R T}(11)}
$$

Where $\eta$ denotes viscosity, $T$ is temperature in Kelvin and $\eta_{0}$ and $E_{\eta}$ indicate variable parameters whose values are provided in Table 2.

\subsubsection{Temperature dependent variation of refractive index for NADES}

The refractive index of all NADES in the temperature range of $(293-323 \mathrm{~K})$ is represented in (Fig. 1 (C)). A linear reduction of the refractive index of all the eutectic mixtures at higher temperature follows a similar pattern to the density. This is due to the increase in free volume due to the destabilization of intermolecular interaction resulting in a lower refractive index. As observed in our previous study, the ascending order of the refractive index is identical to the order of density for NADES under investigation 40. A higher refractive index reduces the free volume and the solubility of target metabolite into the extraction phase thus remain unsuitable for extractive fermentation. NADES with a lower refractive index lags intermolecular interaction hence does not exhibit the property of the aqueous two phase

formation ${ }^{41}$. Hence, NADES with a moderate refractive index value remains a suitable choice for the extraction of naringinase from culture. The observed association of refractive index with temperature is represented in the following equation

$$
n_{D}=s T+u(12)
$$

Where $s$ and $u$ are linearity coefficients, $T$ is the temperature in kelvin and $n_{D}$ represents the refractive index of medium. The values of linearity coefficients are given in Table 2 .

\subsection{Effect of choice of salt and NADES on extraction of narringinase}

The phase formation ability of all NADESs under investigation was observed to be variable depending on the choice of salt in extractive fermentation. The influence of these salts on the phase ratio depends on its corresponding salting out capacity. It could be noted from the results that individual salt in combination with various NADES shows a diverse phase volume ratio in extractive fermentation ${ }^{42}$. Among the various salts considered for investigation, the best phase formation capability was exhibited by sodium sulphate with a 0.38 phase ratio as given in Fig. $2(A)$. This is because of the high dissociation constant and moderate ionic density of the NADES-salt combination which yields maximum a phase ratio. In contradiction, $\mathrm{K}_{2} \mathrm{HPO}_{4}$ exhibits the lowest phase formation capacity even with NADES of moderate density. This is due to the low ionic strength which results in the consumption of salt in large volume for phase formation thus expanding the working volume leading to an infinite dilution of the phase forming components. 
It is observed that the choice of NADESs plays a pivotal role in the investigation of phase formation. Better partition coefficient was observed with NADESs with moderate density. Observation indicates that NADES with low density was observed to have lowest partition coefficient due to its reduced salting out capability Fig. 2 (B). Also, NADES with high density was observed to exhibit low phase partitioning due to the densely packed top phase infiltrating water and target analyte to bottom phase ${ }^{43}$. The phase formation mainly depends on various factors of NADESs including their moderate density and corresponding saturation level. The partition coefficient of the BMC: $\mathrm{X}$ was noted to be 2.1

\subsection{Screening of complex media for naringinase production}

Among the various complications faced in industrial production of naringinase, one of the major cost influence factors involves utilizing complex by-products as low-cost alternatives. Tofu wastewater supported the maximum production of active naringinase enzyme $(247 \mathrm{U} / \mathrm{mL})$ compared to molasses $(123 \mathrm{U} / \mathrm{mL})$ and corn steep liquor $(113 \mathrm{U} / \mathrm{mL})$ (Table 3). Tofu wastewater, a by-product from soy meal industry is rich in minerals and nutrients thus could be exploited for the production of valuable industrial products. Generally, fast metabolizing carbon source does not support naringinase production. Among various carbon sources, molasses and corn steep liquor showed moderate naringinase production from B.amloliquefaciens due to the presence of simple and easily digestible polysaccharide (starch) that promotes facile secondary metabolism. Apart from being a rich source of nitrogen, tofu wastewater is inherent in certain trace elements such as $\mathrm{Mg}, \mathrm{Ca}, \mathrm{Zn}, \mathrm{S}, \mathrm{Cu}, \mathrm{Mn}$ which promotes average growth and high secondary metabolite production. Further, tofu wastewater exhibits slow release of nitrogen which intensifies naringinase production throughout the batch hour.

Table 3

Screening of various low-cost substrates and their corresponding amount of naringinase activity obtained

\begin{tabular}{|lll|}
\hline S.no & Substrate & Enzyme activity $(\mathrm{U} / \mathrm{mL})$ \\
\hline $\mathbf{1}$ & Cane molasses & 123 \\
$\mathbf{2}$ & Corn steep liquor & 113 \\
$\mathbf{3}$ & Soy milk & 247 \\
\hline
\end{tabular}

\subsection{Optimization of naringinase production}

Bacilus amyloliquefaciens was chosen as an organism under investigation for the integrated process production. The extractive fermentation model was constructed and aqueous two phase system with NADES was applied for analysis. High influence was shown by all independent variables that are chosen to be optimized for the recovery of naringinase. The system having BMC: $X(72.5 \% \mathrm{~W} / \mathrm{v})$ and $\mathrm{Na}_{2} \mathrm{SO}_{4}(15 \%$ $\mathrm{w} / \mathrm{v}$ ) gave higher yield. From (Fig. 3) the major influential factors seem to be concentration of NADES and 
tofu wastewater. With $72.5 \%$ DES concentration, $15 \%$ salt concentration, $1.5 \%(\mathrm{w} / \mathrm{v})$ nitrogen source, $249.6 \mathrm{U} / \mathrm{ml}$ of optimum enzyme activity was achieved. Increased yield of $2856.50 \mathrm{IU} / \mathrm{ml}$ was observed from earlier investigation of extractive fermentation for alkaline protease with polyethylene glycol which posseses trivial problems during processing of enzyme ${ }^{44}$. In another investigation, a moderate protease yield was obtained $(1068 \mathrm{U} / \mathrm{ml})$ with PEG and potassium phosphate as extraction medium ${ }^{45}$. In yet another investigation of three phase partitioning of naringinase, a maximal revovery of enzyme was observed to be $719.6 \mathrm{U} / \mathrm{ml}^{17}$. Concentration of $\mathrm{Na}_{2} \mathrm{SO}_{4}$ has significant effect in process of phase separation but shows negligible role in infiltration of active enzyme. The partition of phases is regulated with salt concentration through changing potential difference between two phases. With optimization of these influential factors, naringinase production by extractive fermentation with effective yeild of 249.6 $\mathrm{IU} / \mathrm{ml}$ was achieved.

\subsection{Scale up operation and enzyme recovery}

The scaled up operation of extractive bioconversion for naringinase production provides significantly higher yield compared to the flask level fermentation at identical conditions of optimization. The fermentation done at $30^{\circ} \mathrm{C}$ with $50 \%$ dissolved oxygen at $1.5 \mathrm{~L}$ volume of fresh media provided maximal recovery of enzyme at less batch hours than conventional method. Extractive fermentation accomplished with addition of phase forming components provided significant increase in recovery ( 1.5 fold higher) than the flask level fermentation yielding $344 \mathrm{U} / \mathrm{ml}$ of enzyme in batch mode. This indicates scale up operation for extractive fermentation of naringinase with NADES is possible without affecting the cell viability to greter extent.

\subsection{NADES regeneration for sequential extraction of naringinase}

The extract phase rich in NADES along with the product was removed and NADES was regenerated by adding $\mathrm{NaCl}$ to the mixture. While the product is stripped out from the solvent in to the salt rich raffinate, NADES is left to concentrate as a separate phase on the top. This recycled NADES devoid of naringinase could be reused in another batch extraction process ${ }^{46}$. The amount of NADES recovered in each cycle of extraction signifies that a major part of NADES is being recovered and regenerated. It could be observed clearly from (Fig. 4) that the first cycle of extraction results in $97 \%$ recovery. Subsequent cycles have notable loss of NADES which might be due to the loss of hydrogen bonds which hold the NADES together. This is the result of increased hydration of NADES on successive usage. Solvent regeneration on fifth time results in only $78 \%$ recovery. Further usage has resulted in overdilution of solvent making it unsuitable for phase formation. Additionally, multiple cycles of reutilizing recovered NADES makes it to loose its selectivity for naringinase extraction becoming unsuitable for further exploitation.

\subsection{Repetitive batch fermentation and its effect on recovery}


Repeated batch extractive bioconversion with recycle of raffinate phase rich in $B$. amyloliquefaciens cells potential for carriying out another batch of fermentation was accomplished. Addition of fresh media promotes the production of naringinase with less batch hour for recovery of enzyme. Recycled solvent was supplemented with low amount of fresh NADES for recovery of enzyme ${ }^{47}$. It could be observed that the cloud point was achieved with approximately same volume of NADES as in batch operation. Further, five consecutive batch operation were conducted and the amount of naringinase recovered was identical for all the batch process proving the selectivity of NADES over enzyme. The enzyme activity of all the batch remained close to the optimized range of $300-340 \mathrm{U} / \mathrm{ml}$ ensuring the viability of cells even after repeated operation for third time. Thus, this sequential mode of extractive fermentation provides enhanced recovery of secondary metabolite in an sustainable environment facilitating effective usage of single batch operation.

\subsection{Quantification of enzyme by gel filtration chromatography:}

The concentrated top phase obtained from aqueous two phase extraction were purified by using Size exclusion chromatography. In this study, A $5 \mathrm{ml}$ Sephadex G-15 column was used for fractionation and quantification of the protein. In this process, larger molecules were obtained at the early phase of elution whereas the smaller molecules were obtained at the last phase of elution ${ }^{48}$. Initially the column was equilibrated with $20 \mathrm{mM}$ phosphate buffer $(\mathrm{pH} \mathrm{7.0)}$ ) and the flow rate was maintained at $1 \mathrm{ml} / \mathrm{min}$. The retention volume of the eluents was observed as 17 minutes (Fig. 5 (A)). The number of theoretical plates for the system and buffer were found to be 213. The resolution factor $\left(R_{s}\right)$ between two successive peaks was obtained for the purified fractions through $5 \mathrm{~mL}$ Sephadex G-15 column was observed as 2.73.

\subsection{Ultrapurification of naringinase by anion exchange chromatography:}

The fractions obtained from size exclusion chromatography was subjected to ion exchange chromatography for further purification of the protein sample. The column used in this study is DEAEsepharose anion exchange column with $5 \mathrm{ml}$ bed volume. The column was equilibrated with $20 \mathrm{mM}$ phosphate buffer $(\mathrm{pH} 5.5)$ at a low flow rate of $1 \mathrm{~mL} / \mathrm{min}$. The fractions collected from gel filtration chromatography were passed through the column along with the binding buffer ${ }^{32}$. The molecules showing highest affinity binds tightly to the column. Then the column is supplied with washing buffer to elute out the undesired molecules showing lowest affinity towards the column. Fractionation of bound protein molecule was done by passing a gradient formed with increasing concentration of elution buffer

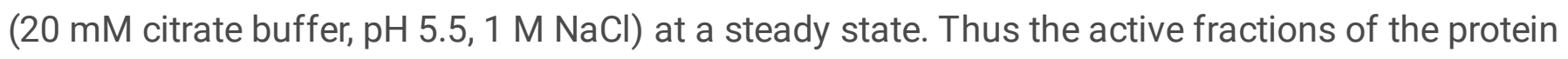
molecule showing the maximum value of absorbance was collected (Fig. 5 (B)). Then the purified protein molecule was subjected to naringin degrading activity. The yield of naringinase was found to be $53.2 \%$ $\mathrm{w} / \mathrm{w}$. The step wise purification enhancement for ultrapure naringinase is provided in Table 4 denoting the yield of each unit operation. 
Table 4

Increase in Purity fold and corresponding to various unit operations involved in purification of naringinase $(n=3$, mean represented along with standard deviation) $(p<0.05)$

\begin{tabular}{|lllll|}
\hline Downstream process & $\begin{array}{l}\text { Total protein } \\
(\mathbf{m g} / \mathbf{m l})\end{array}$ & $\begin{array}{l}\text { Specific activity } \\
(\mathbf{U} / \mathbf{m g})\end{array}$ & $\begin{array}{l}\text { Yield } \\
(\%)\end{array}$ & Purity Fold \\
\hline Extractive fermentation & 417.54 & 55.4 & 100 & 1 \\
\hline Backward extraction & 390.5 & 249.60 & 93.6 & 4.5 \\
\hline Gel filtration chromatography & 328.6 & 736.85 & 78.6 & 13.6 \\
\hline Anion exchange chromatography & 221.8 & 3516.73 & 53.2 & 63.5 \\
\hline
\end{tabular}

\subsection{Enzyme kinetics}

The line-weaver burk plot along with the michaelis menton constants of the reaction were determined. The $K_{m}$ and $V_{\max }$ were found to be $1.5 \times 10^{-3} \mathrm{mmol} \mathrm{min}^{-1}$ and $2.76 \mu \mathrm{mol} / \mathrm{min}$ respectively ${ }^{49}$. The lower $\mathrm{K}_{\mathrm{m}}$ represents that the enzyme reaches its saturation activity in less concentration of substrate itself ${ }^{43}$. And this lower $\mathrm{K}_{\mathrm{m}}$ and higher $\mathrm{V}_{\text {max }}$ specificity of the active site in the enzyme towards the specific residues of the substrate, which could prove the nativity enzyme during partial extraction using natural deep eutectic solvents. The concentration of the enzyme at the active sites $\left[E_{0}\right]$ was calculated to be $0.67 \times 10^{-6} \mu \mathrm{mol}(\mathrm{Fig} .6(\mathrm{~A}))$. The turn over constant $\left(\mathrm{K}_{\mathrm{cat}}\right)$ was found to be $6.86 \times 10^{4}$, which makes the increased catalytic efficiency of $4.57 \times 10^{7} \mathrm{~S}^{-1} \mathrm{~m}^{-1}$. So it can be claimed that the affinity of the produced enzyme toward the substrate is larger when the enzyme is extracted with NADES by extractive fermentation method.

\subsection{Quantitative debittering activity of ultrapure naringinase}

The bitterness of grape juice post treatment with naringinase is greately reduced due to the hydrolysis of key metabolites (naringin and limonin) present earlier in the juice. Post treatment of fresh grape juice with ultrapure naringinase, the colour of the juice became pale due to the bioconversion of naringin to pruning 50 . Fig. (6(B)) denotes the corresponding degree of hydrolysis for naringin and limonin with naringinase enzyme. It could be observed that $23 \%(\mathrm{w} / \mathrm{w})$ of naringin and $64.3 \%(\mathrm{w} / \mathrm{w})$ of limonin is hydrolysed that significantly reduces the bittering activity of grape fruit juice.

\section{Conclusion}

The present investigation is first of its type for extractive bioconversion of tofu wastewater to produce naringinase. Low cost complex media is used for production in this study which drastically reduces the 
production cost of commercial enzyme. Process integration over extractive fermentation reduces the number of unit operation and provides enhanced purity for naringinase in single stage due to the selectivity of the NADES in extraction. Importance of physical parameter evaluation for tailor made solvents could also be observed to modify the extraction agent towards extractive fermentation. Batch operation for recovery of naringinase is optimized and maximal recovery of $249.7 \mathrm{U} / \mathrm{ml}$ of enzyme is obtained. Further, the process is scaled up with a desk top custom made bioreactor of capacity $3 \mathrm{~L}$ and repeated sequence of extraction and fermentation in fed batch mode is operated for evaluation of efficiency of operation. The enzyme obtained was quantified with preparative chromatography which provides facile industrial scale analysis of naringinase recovery. Additionally, debittering activity of ultrapure enzyme fraction obtained from anion exchange chromatography is evaluated agiants grape fruit juice. Thus, an industrial model of extractive bioconversion of naringinase using task specific cost effective solvent for naringinase production is proposed by this investigation.

\section{Declarations}

\section{Acknowledgement:}

Senthilkumar Rathnasamy is grateful for the support provided by the Department of Science and Technology, India for providing research grant through SERB (EMR/2016/003669) to accomplish the investigation. Harishbabu Balaraman is grateful for the CSIR-SRF (Direct) fellowship provided by the Council of Scientific \& Industrial Research, India to accomplish the investigation. The authors also acknowledge Bio separation laboratory, School of Chemical and Biotechnology, SASTRA Deemed to be University, Thanjavur, Tamil Nadu - 613 401, India for providing the chromatography facility.

\section{Conflict of Interest:}

The authors have no conflict of interest towards any individual (or) educational and research institution of any kind. The authors further claim that these research findings described herein are their own proposals.

\section{Availability of Data and Materials:}

All data generated or analyzed during this study are included in this published article [and its supplementary information files].

\section{References}

1. Puri, M., Banerjee, A. \& Banerjee, U. J. P. B. Optimization of process parameters for the production of naringinase by Aspergillus niger MTCC 1344. 40, 195-201 (2005).

2. Chang, H.-Y. et al. Purification and characterisation of Aspergillus sojae naringinase: the production of prunin exhibiting markedly enhanced solubility with in vitro inhibition of HMG-CoA reductase. 124, 234-241 (2011). 
3. Zhang, T., Yuan, W., Li, M., Miao, M. \& Mu, W. Purification and characterization of an intracellular a-lrhamnosidase from a newly isolated strain, Alternaria alternata SK37.001. Food Chemistry 269, 6369, doi:https://doi.org/10.1016/j.foodchem.2018.06.134 (2018).

4. Awad, G. E., El Aty, A. A. A., Shehata, A. N., Hassan, M. E. \& Elnashar, M. M. J. B. Covalent immobilization of microbial naringinase using novel thermally stable biopolymer for hydrolysis of naringin. 6, 14 (2016).

5. Zhu, Y. et al. Purification and characterization of a naringinase from a newly isolated strain of Bacillus amyloliquefaciens 11568 suitable for the transformation of flavonoids. 214, 39-46 (2017).

6. Ribeiro, M. H. Naringinases: occurrence, characteristics, and applications. Applied Microbiology and Biotechnology 90, 1883-1895, doi:10.1007/s00253-011-3176-8 (2011).

7. Puri, M. \& Kalra, S. Purification and characterization of naringinase from a newly isolated strain of Aspergillus niger 1344 for the transformation of flavonoids. World Journal of Microbiology and Biotechnology 21, 753-758, doi:10.1007/s11274-004-5488-7 (2005).

8. Gangadharan, D., Sivaramakrishnan, S., Nampoothiri, K. M., Sukumaran, R. K. \& Pandey, A. Response surface methodology for the optimization of alpha amylase production by Bacillus amyloliquefaciens. Bioresource Technology 99, 4597-4602, doi:https://doi.org/10.1016/j.biortech.2007.07.028 (2008).

9. Sandhya, C., Sumantha, A., Szakacs, G. \& Pandey, A. Comparative evaluation of neutral protease production by Aspergillus oryzae in submerged and solid-state fermentation. Process Biochemistry 40, 2689-2694, doi:https://doi.org/10.1016/j.procbio.2004.12.001 (2005).

10. Shanmugaprakash, M., Kirthika, J., Ragupathy, J., Nilanee, K. \& Manickam, A. Statistical based media optimization and production of naringinase using Aspergillus brasiliensis 1344. International Journal of Biological Macromolecules 64, 443-452, doi:https://doi.org/10.1016/j.jibiomac.2013.12.033 (2014).

11. Zhu, Y. et al. Characterization of a naringinase from Aspergillus oryzae 11250 and its application in the debitterization of orange juice. Process Biochemistry 62, 114-121, doi:https://doi.org/10.1016/j.procbio.2017.07.012 (2017).

12. Puri, M., Banerjee, A. \& Banerjee, U. C. Optimization of process parameters for the production of naringinase by Aspergillus niger MTCC 1344. Process Biochemistry 40, 195-201, doi:https://doi.org/10.1016/j.procbio.2003.12.009 (2005).

13. Borkar, V., Chakraborty, S. \& Gokhale, J. S. Fermentative Production of Naringinase from Aspergillus niger van Tieghem MTCC 2425 Using Citrus Wastes: Process Optimization, Partial Purification, and Characterization. Applied Biochemistry and Biotechnology, doi:10.1007/s12010-020-03385-9 (2020).

14. Puri, M., Kaur, A., Barrow, C. J. \& Singh, R. S. Citrus peel influences the production of an extracellular naringinase by Staphylococcus xylosus MAK2 in a stirred tank reactor. Applied Microbiology and Biotechnology 89, 715-722, doi:10.1007/s00253-010-2897-4 (2011).

15. Vandar, V., S, S. \& Rathnasamy, S. Lactoperoxidase Extraction from Caprine Milk using Conventional and Ionic Liquid Based ATP System. International Journal of ChemTech Research 6, 974-4290 
(2014).

16. Chen, Y. et al. Purification and Characterization of a Naringinase from Aspergillus aculeatus JMUdb058. Journal of Agricultural and Food Chemistry 61, 931-938, doi:10.1021/jf303512q (2013).

17. Shanmugaprakash, M., Vinothkumar, V., Ragupathy, J. \& Reddy, D. A. Biochemical characterization of three phase partitioned naringinase from Aspergillus brasiliensis MTCC 1344. International Journal of Biological Macromolecules 80, 418-423, doi:https://doi.org/10.1016/j.ijbiomac.2015.06.057 (2015).

18. Rathnasamy, S. \& Kumaresan, R. Integration and investigation of metal ligands in aqueous two phase extraction and its partition coefficient studies in papain extraction. (2013).

19. Shanmugaprakash, M., Vinothkumar, V., Ragupathy, J. \& Reddy, D. A. J. I. j. o. b. m. Biochemical characterization of three phase partitioned naringinase from Aspergillus brasiliensis MTCC 1344. 80, 418-423 (2015).

20. Amaral, Y. M. S., da Silva, O. S., de Oliveira, R. L., Porto, T. S. J. P. B. \& Biotechnology. Production, extraction, and thermodynamics protease partitioning from Aspergillus tamarii Kita UCP1279 using PEG/sodium citrate aqueous two-phase systems. 1-8 (2020).

21. Sales, A. E. et al. Integrated process production and extraction of the fibrinolytic protease from Bacillus sp. UFPEDA 485. 170, 1676-1688 (2013).

22. Babu Balaraman, H., Sivasubramanian, A. \& Kumar Rathnasamy, S. Sustainable valorization of meat processing wastewater with synergetic eutectic mixture based purification of R-Phycoerythrin from porphyrium cruentium. Bioresource Technology 336, 125357, doi:https://doi.org/10.1016/j.biortech.2021.125357 (2021).

23. Cunha, S. C. \& Fernandes, J. O. Extraction techniques with deep eutectic solvents. TrAC Trends in Analytical Chemistry 105, 225-239, doi:https://doi.org/10.1016/j.trac.2018.05.001 (2018).

24. Bosiljkov, T. et al. Natural deep eutectic solvents and ultrasound-assisted extraction: Green approaches for extraction of wine lees anthocyanins. Food and Bioproducts Processing 102, 195203, doi:https://doi.org/10.1016/j.fbp.2016.12.005 (2017).

25. Balaraman, H. B. \& Rathnasamy, S. K. J. M. J. Selective purification of protease from ginger and sodom apple by ultrasound assisted liquid-liquid microextraction using natural deep eutectic solvent. 150, 104132 (2019).

26. Balaraman, H. B. \& Rathnasamy, S. K. High selective purification of IgY from quail egg: Process design and quantification of deep eutectic solvent based ultrasound assisted liquid phase microextraction coupled with preparative chromatography. International Journal of Biological Macromolecules 146, 253-262, doi:https://doi.org/10.1016/j.ijbiomac.2019.12.242 (2020).

27. Farajzadeh, M. A., Mohebbi, A., Pazhohan, A., Nemati, M. \& Afshar Mogaddam, M. R. Air-assisted liquid-liquid microextraction; principles and applications with analytical instruments. TrAC Trends in Analytical Chemistry 122, 115734, doi:https://doi.org/10.1016/j.trac.2019.115734 (2020).

28. Afshar Mogaddam, M. R., Mohebbi, A., Pazhohan, A., Khodadadeian, F. \& Farajzadeh, M. A. Headspace mode of liquid phase microextraction: A review. TrAC Trends in Analytical Chemistry 110, 
8-14, doi:https://doi.org/10.1016/j.trac.2018.10.021 (2019).

29. Habelt, K. \& Pittner, F. A rapid method for the determination of naringin, prunin, and naringenin applied to the assay of naringinase. Analytical Biochemistry 134, 393-397, doi:https://doi.org/10.1016/0003-2697(83)90314-7 (1983).

30. Torabizadeh, H. \& Mikani, M. J. I. j. o. b. m. Kinetic and thermodynamic features of nanomagnetic cross-linked enzyme aggregates of naringinase nanobiocatalyst in naringin hydrolysis. 119, 717-725 (2018).

31. Romero, C., Manjón, A., Bastida, J. \& Iborra, J. A method for assaying the rhamnosidase activity of naringinase. Analytical Biochemistry 149, 566-571, doi:https://doi.org/10.1016/00032697(85)90614-1 (1985).

32. Karthiraj, T., Harish Babu, B. \& Senthil Kumar, R. Task-specific deep eutectic solvent based extraction coupled cascade chromatography quantification of a-glucosidase inhibitory peptide from Ocimum tenuriflorum seeds. Microchemical Journal 157, 104883, doi:https://doi.org/10.1016/j.microc.2020.104883 (2020).

33. Rathnasamy, S. K., sri Rajendran, D., Balaraman, H. B. \& Viswanathan, G. J. A. R. Functional deep eutectic solvent-based chaotic extraction of phycobiliprotein using microwave-assisted liquid-liquid micro-extraction from Spirulina (Arthrospira platensis) and its biological activity determination. 44, 101709 (2019).

34. Ribeiro, I. A. \& Ribeiro, M. H. L. Naringin and naringenin determination and control in grapefruit juice by a validated HPLC method. Food Control 19, 432-438, doi:https://doi.org/10.1016/j.foodcont.2007.05.007 (2008).

35. Balaraman, H. B., Sivasubramaniyam, A. \& Rathnasamy, S. K. High selective purification of Quercetin from Peanut hull using protic deep eutectic mixture based liquid-liquid microextraction. Microchemical Journal 152, 104444, doi:https://doi.org/10.1016/j.microc.2019.104444 (2020).

36. Rathnasamy, S. K., Rajendran, D. S., Balaraman, H. B. \& Viswanathan, G. Functional deep eutectic solvent-based chaotic extraction of phycobiliprotein using microwave-assisted liquid-liquid microextraction from Spirulina (Arthrospira platensis) and its biological activity determination. Algal Research 44, 101709, doi:https://doi.org/10.1016/j.algal.2019.101709 (2019).

37. Babu Balaraman, H., Viswanathan, G., Muniasamy, R., Gayatri, T. \& Kumar Rathnasamy, S. Sustainable valorization of papaya peels for thrombolytic cysteine protease isolation by ultrasound assisted disruptive liquid phase microextraction with task specific switchable natural deep eutectic solvents. Microchemical Journa/ 175, 107118, doi:https://doi.org/10.1016/j.microc.2021.107118 (2022).

38. Balaraman, H. B., Rathnasamy, S. K. J. J. o. C. \& Data, E. Thermophysical and molar volume aberration of amphiphilic eutectic mix of bivalent diols and ammonium-ionic liquid. 64, 3307-3315 (2019).

39. Balaraman, H., Selvasembian, R., Rangarajan, V. \& Rathnasamy, S. Sustainable and Green Engineering Insights on Deep Eutectic Solvents toward the Extraction of Nutraceuticals. ACS 
Sustainable Chemistry \& Engineering 9, 11290-11313, doi:10.1021/acssuschemeng.1c03034 (2021).

40. Balaraman, H. B., Sivasubramaniyam, A. \& Rathnasamy, S. K. High selective purification of Quercetin from Peanut hull using protic deep eutectic mixture based liquid-liquid microextraction. Microchemical Journal, 104444, doi:https://doi.org/10.1016/j.microc.2019.104444 (2019).

41. Balaraman, H. B. \& Rathnasamy, S. K. Thermophysical and Molar Volume Aberration of Amphiphilic Eutectic Mix of Bivalent Diols and Ammonium-lonic Liquid. Journal of Chemical \& Engineering Data 64, 3307-3315, doi:10.1021/acs.jced.9b00134 (2019).

42. Rathnasamy, S. K. et al. One-pot simultaneous production and sustainable purification of fibrinolytic protease from Bacillus cereus using natural deep eutectic solvents. Scientific Reports 10, 13356, doi:10.1038/s41598-020-70414-2 (2020).

43. Balaraman, H. B. \& Rathnasamy, S. K. Kinetics and microwave-assisted extractive transesterification studies of high octane methyl esters (HOME) from karanja and chicken lard oil using protic deep eutectic solvent. Fuel 268, 117299, doi:https://doi.org/10.1016/j.fuel.2020.117299 (2020).

44. Pandey, S. K. \& Banik, R. M. Extractive fermentation for enhanced production of alkaline phosphatase from Bacillus licheniformis MTCC 1483 using aqueous two-phase systems. Bioresource Technology 102, 4226-4231, doi:https://doi.org/10.1016/j.biortech.2010.12.066 (2011).

45. Hotha, S., Banik, R. M. J. J. o. C. T., Biotechnology: International Research in Process, E. \& Technology, C. Production of Alkaline Protease by Bacillus thuringiensis H 14 in Aqueous Two-Phase Systems. 69, 5-10 (1997).

46. Wang, $\mathrm{H}$. et al. Extraction of cellulose nanocrystals using a recyclable deep eutectic solvent. Cellulose 27, 1301-1314, doi:10.1007/s10570-019-02867-2 (2020).

47. Rathnasamy, S. K., Balaraman, H. B. \& Muniasamy, R. Air-assisted dispersive liquid phase microextraction coupled chromatography quantification for purification of therapeutic lectin from aloe vera - A potential COVID-19 immune booster. Microchemical Journal 165, 106187, doi:https://doi.org/10.1016/j.microc.2021.106187 (2021).

48. Rathnasamy, S. \& Debora, J. Extraction and Purification of C-Phycocyanin from Spirulina platensis Using Aqueous Two Phase Extraction and its Applications. Asian Journal of Chemistry 26, 37293732, doi:10.14233/ajchem.2014.17063 (2014).

49. Huang, W. et al. Controllable immobilization of naringinase on electrospun cellulose acetate nanofibers and their application to juice debittering. International Journal of Biological Macromolecules 98, 630-636, doi:https://doi.org/10.1016/j.jjbiomac.2017.02.018 (2017).

50. Puri, M. \& Banerjee, U. C. Production, purification, and characterization of the debittering enzyme naringinase. Biotechnology Advances 18, 207-217, doi:https://doi.org/10.1016/S07349750(00)00034-3 (2000).

\section{Figures}




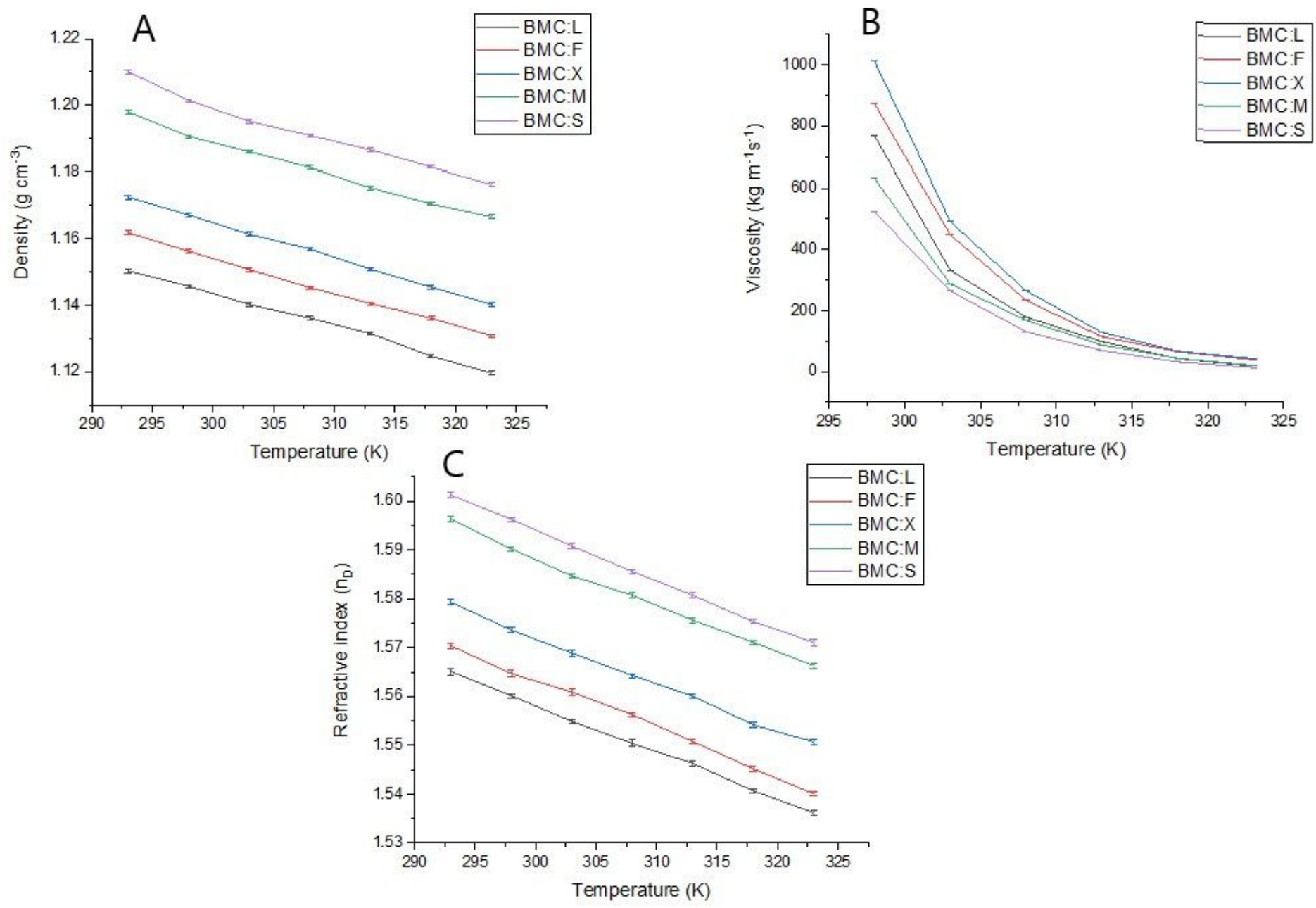

\section{Figure 1}

Temperature dependence (273-373 K) of Density (A), Viscosity (B) and Refractive index (C) for all NADES formed by combining Benzyl trimethyl ammonium chloride (BMC:L) with Lactose , Fructose, Xylose, Maltose and Sucrose in temperature range of $273-323 \mathrm{~K}$

\section{Figure 2}

The influence of NADES and salt type on the phase ratio (A) and partition coefficient (B) for naringinase obtained through extractive fermentation. The yield differs based on the choice of NADES and salt proves the selectivity of the system towards naringinase

\section{Figure 3}

Response surface plot showing the cumulative effect of concentration of (A) $\mathrm{Na}_{2} \mathrm{SO}_{4}$ with NADES (B) soy milk with NADES and (C) soy milk with $\mathrm{Na}_{2} \mathrm{SO}_{4}$ on extractive fermentation of naringinase. 


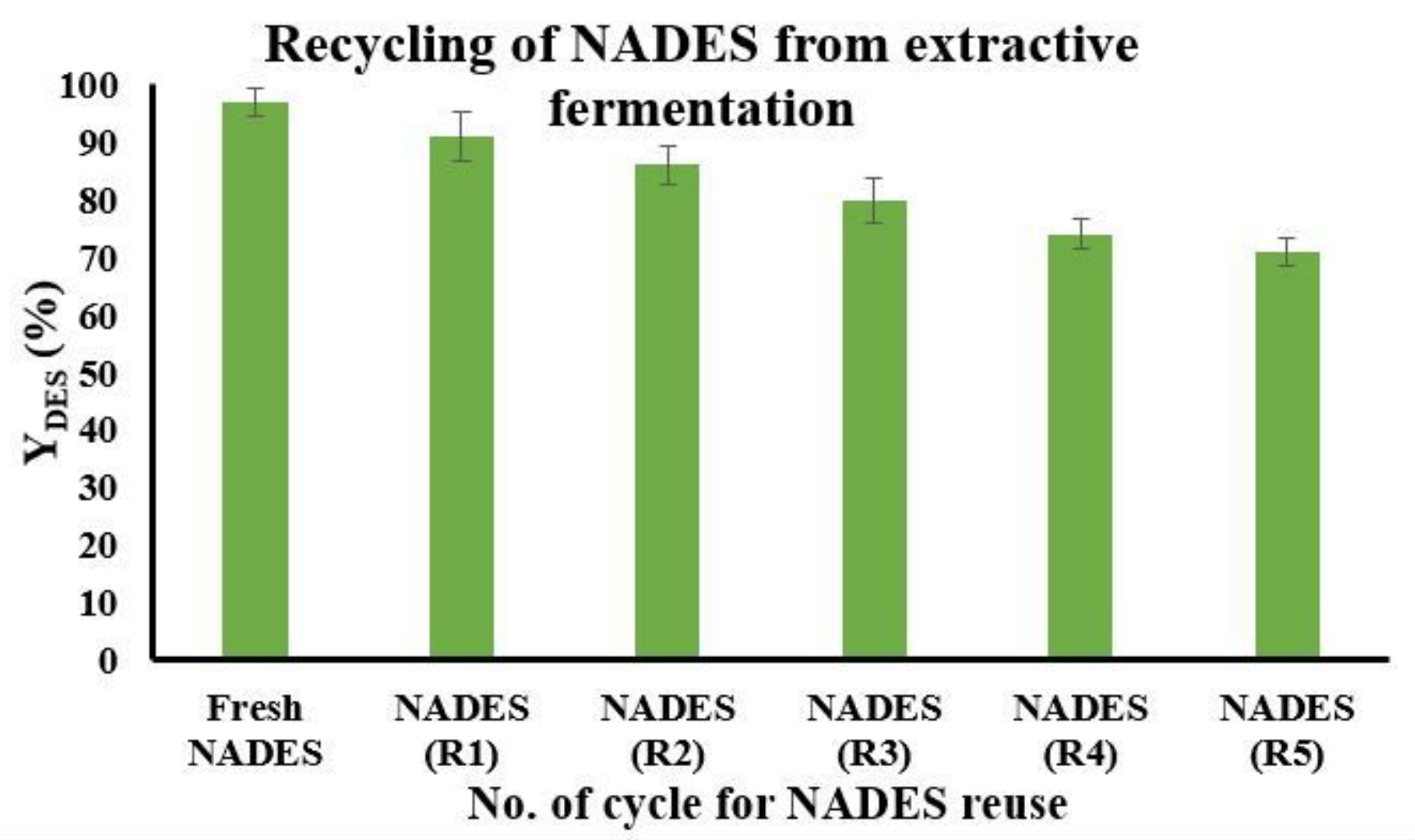

Figure 4

Recycling ability of NADES is determined for 5 cycles. It is observed that the amount of NADES recovered reduces significantly after third cycle denoting loss of selective partitioning and extraction ability. 


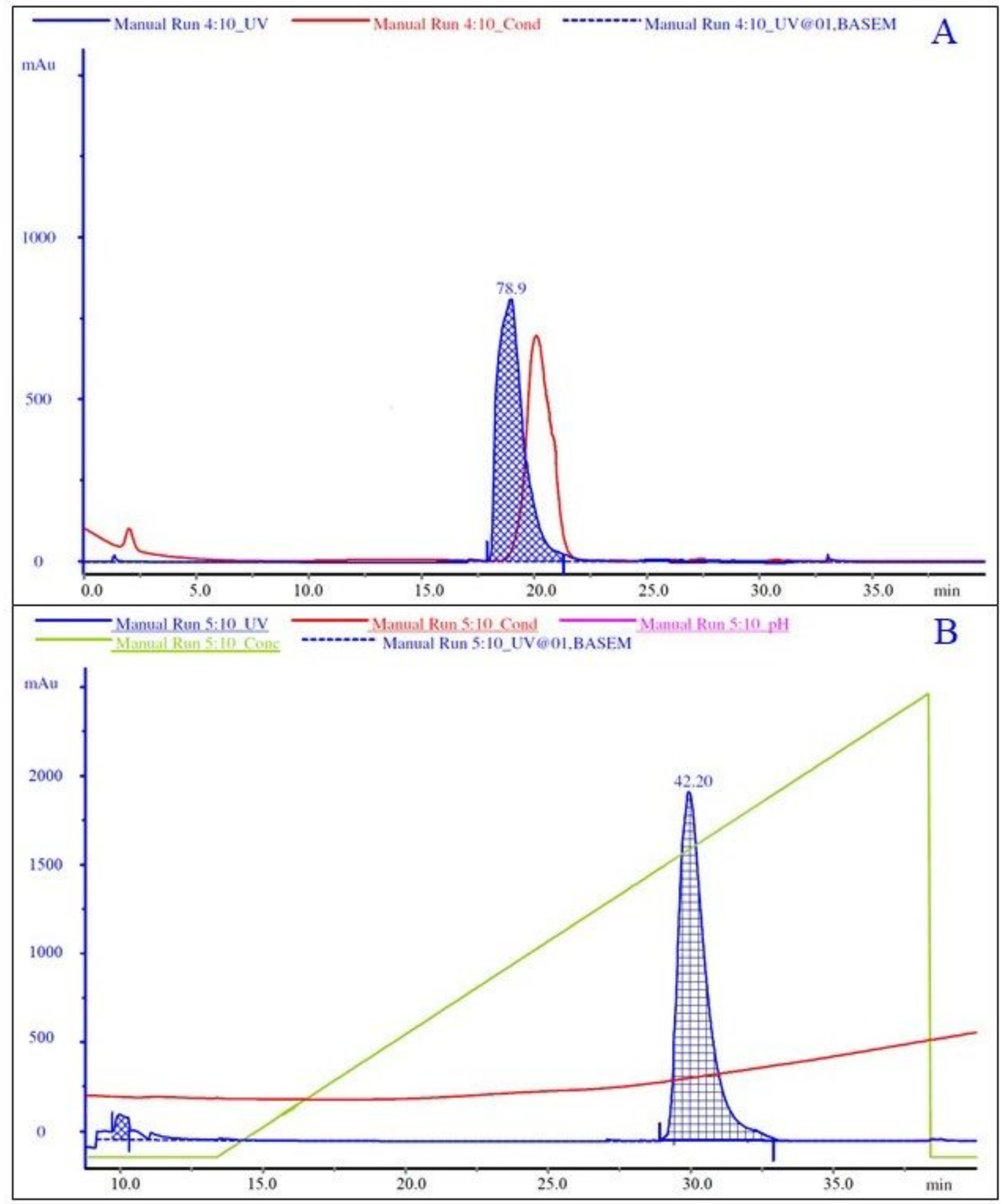

Figure 5

Chromatogram representing purification of naringinase by (A) Size exclusion chromatography and (B) anion exchange chromatography denoting the equillibriation and elution profiles with their respective buffers. The blue line indicates response from UV detector and red line indicates conductivity response 
Figure 6

Graphical plots representing (A) kinetics of specific enzyme activity of naringinase with naringin as substrate (B) debittering activity of ultrapure fraction of enzyme in grape juice

\section{Supplementary Files}

This is a list of supplementary files associated with this preprint. Click to download.

- Supplementarymaterial.docx 